\title{
Asymmetric Conditional Volatility in International Stock Markets
}

\author{
Nuno B. Ferreira*, Rui Menezes* and Diana A. Mendes* \\ *Department of Quantitative Methods, IBS - ISCTE Business School, ISCTE, Av. \\ Forcas Armadas, 1649-025 Lisboa, Portugal, e-mail: nuno.ferreira@iscte.pt
}

\begin{abstract}
Recent studies show that a negative shock in stock prices will generate more volatility than a positive shock of similar magnitude. The aim of this paper is to appraise the hypothesis under which the conditional mean and the conditional variance of stock returns are asymmetric functions of past information. We compare the results for the Portuguese Stock Market Index PSI 20 with six other Stock Market Indices, namely the SP 500, FTSE 100, DAX 30, CAC 40, ASE 20, and IBEX 35. In order to assess asymmetric volatility we use autoregressive conditional heteroskedasticity specifications known as TARCH and EGARCH. We also test for asymmetry after controlling for the effect of macroeconomic factors on stock market returns using TAR and M-TAR specifications within a VAR framework. Our results show that the conditional variance is an asymmetric function of past innovations raising proportionately more during market declines, a phenomenon known as the leverage effect. However, when we control for the effect of changes in macroeconomic variables, we find no significant evidence of asymmetric behaviour of the stock market returns. There are some signs that the Portuguese Stock Market tends to show somewhat less market efficiency than other markets since the effect of the shocks appear to take a longer time to dissipate.
\end{abstract}

Key words: Asymmetric conditional volatility, stock market returns, threshold adjustment, vector autoregression

\section{Introduction}

It is well known in the financial literature that stock market index returns are positively autocorrelated, especially when dealing with high frequency data. This fact has been often attributed to non-synchronous trading phenomena (see e.g. Fischer [1], Scholes and Williams [2], and Lo and Mackinlay [3]), and to time-varying short-term expected returns, or risk premia in the sense of 
Fama and French [4], and Conrad and Kaul [5]. It is also acknowledged in the empirical literature that the unconditional distribution of returns appears to be excessively leptokurtic for many stock market index prices. Hamao et al.[6], and Wei et al.[7], for example, found the presence of excess kurtosis in the normalized residuals in virtually all mature and emerging stock markets. Mandelbrot [8], and Fama [9] define excess kurtosis when the return series exhibit the fat-tailed feature and leptokurtosis when there is observable greater excess kurtosis relatively to the normal distribution. Leptokurtosis may pose a number of problems when trying to analyse empirically the dynamic behaviour of stock markets. In order to deal with these problems, several authors have attempted to use more general formulations of the distributions used in empirical work (see for example Mandelbrot [8], Fama [9], and Nelson [10]). However, the subject appears to be far from solved in a totally satisfying manner.

There are several papers that are studying these topics by applying different ideas and techniques ([11]; 114; [12]; [15]; [16]; [17]; [18]; [19]).

Another issue obviously related to the previous one concerns the fact that short-term stock returns usually exhibit volatility clustering. Links between information and volatility of financial assets are of course also central to financial economics. Ross [21], for example, argued that volatility could be regarded as a measure of the information flow. Moreover, as noted above, empirical researchers also report significant relationships between flows and volatility.

Volatility clustering and excess kurtosis may arise in the context of asymmetric behaviour of price index movements or their corresponding returns. Such asymmetry may depend on other economic and financial factors or simply be the result of the time-path behaviour of the price index series itself. In the latter case, the conditional variance may be an asymmetric function of past innovations raising proportionately more during market declines. This means that changes in stock prices or returns tend to be negatively related to changes in volatility (see e.g. Black [22], and Christie [23]), i.e. the so-called leverage effect, where stock price declines increase the financial leverage and, consequently, the degree of risk (volatility).

Authors such as Engle [24], inter alia, defend the idea that financial market volatility can be predictable. In order to forecast the volatility, several methods have been proposed in the specialised literature, many of which are based on regression-like models. Clearly, the classical regression model is unsuited when the residuals exhibit features that depart from the basic OLS assumptions. Non-normality, autocorrelation, and heteroskedasticity are some of the problems that are typically present in this type of data. In order to deal with the problems of autocorrelation and heteroskedasticity, ARCH/GARCH specifications are usually well suited. However, they assume that the conditional variance has a symmetric behaviour and thus may not fully capture the issues 
of non-normality. In our context, we shall use TARCH and EGARCH model specifications to assess the extent of non-linear dynamic behaviour underlying the stock prices data of various markets. The models used in this paper belong to special classes of non-linear models that usually generalise the more traditional autoregressive conditional heteroskedasticity models.

Autoregressive conditional heteroskedasticity $(\mathrm{ARCH})$ models have been used quite frequently in applied financial research work. Among the most popular extensions are the Threshold ARCH (TARCH) model proposed by Zakoian [25], and Glosten et al. [26], and the Exponential GARCH (EGARCH) model proposed by Nelson [10]. Engle and $\mathrm{Ng}$ [27] indicate that these latter volatility models usually outperform standard Generalized ARCH (GARCH) models.

An alternative way for modelling the volatility of stock market returns is based on a Vector Autoregressive (VAR) specification using other variables that may help to explain the behaviour of financial markets. In this context we could observe e.g. (Sims [28], [29] and [30]]).

A VAR system is a reduced form model that accounts for the effect of lagged values of the endogenous variables on the dynamic behaviour of the whole system. Each variable is specified as a function of the past values of the other (endogenous) variables but does not deppend on their contemporary values. VAR models are well suited to deal with situations where an error correction mechanism (ECM) can describe the behaviour of the system. In this case a corresponding Vector Error Correction model (VECM) can be specified and fully estimated using ML procedures. This is important when one needs to separate the long-run and short-run components of the dynamic mechanism.

However, there are several criticisms with respect to VAR models. One is the likely loss in efficiency due to lack of apriori information. Another problem relates with the large number of parameters to be estimated as the dynamic structure of the system increases. This is especially relevant for small data sets. Other problems may arise when dealing with impulse response analysys.

Since stock market returns and other macroeconomic variables may all be endogenous in the system, the VAR specification is a quite satisfactory way for modelling the volatility. Testing for asymmetries in this context entails the use of TAR and M-TAR tests of the VAR residuals (see e.g. Enders and Granger [31], and Enders and Siklos [32]). It is important, therefore, to obtain comparative results of the relative performance of these two alternative types of volatility models, and this is the main aim of the present paper. 


\section{Asymmetry and Volatility Models}

The trade-off between risk and expected return is a foundation of modern financial theory, as can be seen in the Capital Asset Pricing Model (CAPM), Arbitrage Pricing Model (APT), Portfolio Theory, and Option Pricing Theory. In all these models, volatility plays a central role in determining the fair value of a security. To quantify the risk level, several researchers have used quantitative statistical indicators, where the standard deviation is one of the most popular measures. This statistical measure, however, assumes a symmetric variation of price levels. Yet, asymmetries are easily observed in time series of financial data, and already constitute an important phenomenon.

For example, there are good reasons to believe that speculative financial asset changes have in general an asymmetric behaviour. As mentioned above, the leverage effect was found in many empirical studies that analyse the behaviour of stock returns. This circumstance highlights the need for using asymmetric models when one is analysing data on stock market behaviour. Asymmetric behaviour in financial data can be detected with relatively ease, since volatility raises more for negative shocks than for positive shock with the same amplitude. In order to account for this phenomenon, two extensions of the basic GARCH model can be used, among other possibilities: the Threshold Autoregressive Conditional Heteroskedasticity (TARCH) and the Exponential Generalised Autoregressive Conditional Heteroskedasticity (EGARCH) models (see Nelson [10], Zakoian [25], and Glosten et al. [26]).

Additionally, this study also investigates the relationship between the volatility terms for index returns and two macroeconomic variables: Dividend Yield (Dy) and the Price Earnings per Share (PER). These variables will be used for TAR

and M-TAR tests based on a VAR model specification as explained above. This approach is similar to that used by Gjerde and Saettem [33] and Rapach [34]. Note that the daily frequency of the stock market returns variable imposes restrictions on the choice and availability of other macroeconomic variables that could also be of relevance but are not available with such a frequency.

\subsection{The Data}

The data used in this study consists of daily stock index closing prices for seven stock market indices over a period from January 1993 until December 2002 (Datastream database). Stock returns were computed as the logarithm of the ratio of the index values at time $t$ and time $t-1$. We consider the following indices: the Standard and Poors 500 (S\&P500) to represent the whole US economy, the CAC 40 index for France, the FTSE 100 for the UK, the DAX 
for Germany, the IBEX 35 for Spain, the ASE 20 for Greece and finally, the PSI 20 for Portugal.

The exchanges of Paris, London, Frankfurt, and New York represent four of the world's major centres for the trading and distribution of both domestic and international equities with special interest for Portuguese investors, and this justifies their inclusion in the analysis. Furthermore, France and Germany are viewed as "core" European economies, being members of the Exchange Rate Mechanism since its inception in 1979, and of the European common currency - the Euro - since 1999. The remaining stock markets are more similar to the Portuguese stock market and we may expect somewhat similar results for the three southern European countries.

\subsection{Methodological Issues and Empirical Results}

The existence of important inter-relationships between the major financial stock markets gives support to the use of VAR systems in the context of the analysis of volatility. There is the need to understand how shocks and volatility in one market are transmitted to other markets. To this end, we need to look at the extent to which multi-lateral interaction exists between these markets. Thus, after identifying the channels of interaction we can observe the implications of volatility co-movements between different markets.

VAR models have been increasingly used in the analysis of the relationships between financial markets. VAR models may be used with both stationary and non-stationary data. In the latter case, they provide a convenient framework to test for cointegration using the Johansen methodology, but the methodology is far more general and is also quite useful when the variables are stationary. On the other hand, ARCH/GARCH type models have been widely used to test hypotheses concerning the conditional volatility of stock market returns. Both methods can be employed to model volatility in the stock market. However, it is important to note that employing these different models, each one measures and captures different types of volatility.

TARCH and EGARCH models are especially adequate to model the volatility measured in terms of error's variance in the context of asymmetry. On the other hand, TAR and M-TAR models systematise the volatility of the dependent variables in a VAR system, captured from the corresponding residuals. The TAR and M-TAR models based on VAR systems are especially designed to capture asymmetric multivariate effects in a multi-equation framework, which certainly constitutes an important advantage over the single-equation multivariate TARCH and EGARCH models. However, one important disadvantage of the former models is that they do not account for conditional heteroskedas- 
ticity that may be present in the data.

The first step of our analysis consists of estimating univariate $\mathrm{TARCH}(1,1)$ and EGARCH $(1,1)$ models in order to assess whether our series exhibit or not some type of conditional volatility asymmetric behaviour. For the TARCH model we use the following specification of the conditional variance:

$$
\sigma_{t}^{2}=\omega+\alpha \varepsilon_{t-1}^{2}+\gamma \varepsilon_{t-1}^{2} d_{t-1}+\beta \sigma_{t-1}^{2}
$$

where the variance $\sigma_{t}^{2}$ is a function of the past squared residuals $\varepsilon_{t-1}^{2}$, and of its own lagged values $\sigma_{t-1}^{2}$. The variable $d_{t-1}$ is a dummy variable equal to one if $\varepsilon_{t-1}>0$, and equal to zero otherwise. $\omega, \alpha, \gamma$, and $\beta$ are the parameters of the conditional variance equation that will be estimated. The equation of the conditional variance for the EGARCH model takes the following form:

$$
\log \sigma_{t}^{2}=\omega+\beta \log \sigma_{t-1}^{2}+\alpha\left|\frac{\varepsilon_{t-1}}{\sigma_{t-1}}\right|+\gamma \frac{\varepsilon_{t-1}}{\sigma_{t-1}}
$$

where the symbols are as described earlier. The results of the univariate TARCH and EGARCH models are reported in Table 1.

As can be seen, the estimates of $\alpha$ are almost all significantly positive, except for the US and UK, where we found no significant $\alpha$ coefficients when the TARCH model was used. In this model, we obtain good news $\left(\varepsilon_{t} \leq 0\right)$ and bad news $\left(\varepsilon_{t}>0\right)$. This means that the model has differential effects on the conditional variance - good news has an impact on $\alpha$, while bad news has an impact on $\alpha+\gamma$. We say that the leverage effect exists if $\gamma \neq 0$ and an asymmetric effect is observed if $\gamma>0$. The leverage effect expressed in $\varepsilon_{t-1}^{2} d_{t-1}$ is associated with the parameter $\gamma$ in equation (1), and at the same time it must reveal statistical significance for both models in Table 1 . In the EGARCH specification, the asymmetric behaviour exists if $\gamma<0$.

Another important result to note is that the estimate of $\beta$ in the EGARCH model is less than one for all the markets, which implies that all the moments of the relevant statistical distribution exist and that the quasi-maximum likelihood estimators are likely to be consistent and asymptotically normal. Thus, relatively to the results reported in Table 1, we may conclude that the leverage effect hypothesis cannot be rejected in any case. Likewise, there is evidence of an asymmetric behaviour of the stock markets for all the countries analysed. 
Table 1. TARCH(1,1) and EGARCH(1,1)

A more detailed analysis of the results reported in Table 1 reveals that the coefficient $\alpha$ for Portugal is higher than the corresponding values for the remaining markets, both for the TARCH and EGARCH model 11 These figures imply that shocks in the Portuguese stock market tend to have longer durations, or higher persistence. As Bala and Prematne [35] state, this circumstance implies that the effect of shocks in earlier periods for Portugal tend to linger around for a longer period than it does in other stock markets. One possible explanation for this result is that it may imply that the Portuguese stock market shows less market efficiency than the other markets analysed, since the effects of shocks take longer time to dissipate.

1 Except for Greece in the EGARCH model, whose value of $\alpha$ is 0.2602 , whereas for Portugal is 0.2349 . 
However, one should note that this does not mean that the Portuguese stock market is not efficient. Our results should just be analysed in relative terms and comparatively with other markets.

We turn now to consider the results of multivariate TARCH and EGARCH models where the Portuguese stock market index is the dependent variable, in order to obtain the OLS residuals, and take the remaining market indices successively as the independent market index for all possible relations established with the Portuguese stock market. These results may bring new information relatively to the results of Table 1 , since it is quite likely that the stock markets establish interactions between them. We restrict our analysis to the interactions between the Portuguese stock market and the remaining markets, successively, and we estimate multivariate TARCH and EGARCH models. The results reported in Table. 2 are very similar to those reported earlier and lead in general to the same conclusions. The estimated coefficients, however, appear now significant in all cases.

We are now going to test the possibility that an adjustment process occurs for different stock market price index combinations with the Portuguese index, which have asymmetric cycles in the respective time series. For this test we employ the methodology of TAR and M-TAR models, where the indices appear as endogenous variables and the macroeconomic factors as control exogenous variables.

The basic starting point for this analysis is the long-run relationship between the relevant variables to the model. The long-run equilibrium relationship between two time series $z_{t}$ and $x_{t}$ can be estimated as a standard regression model $z_{t}=\alpha+\beta x_{t}+\mu_{t}$, where $\alpha$ and $\beta$ are the estimated parameters, and $\mu_{t}$ is a disturbance term that may be serially correlated. The parameter $\beta$ gives the magnitude of adjustment of $z$ to variations in $x$, and is the long-run elasticity of the two variables if they are measured in logs. If $\beta<1$, then shifts in $x$ are not fully passed onto $z$.

The second step of the methodology focuses on the OLS estimates of $\rho_{1}$ and $\rho_{2}$ in the following error correction model:

$$
\Delta \mu_{t}=I_{t} \rho_{1} \mu_{t-1}+\left(1-I_{t}\right) \rho_{2} \mu_{t-1}+\varepsilon_{t}
$$

where $\varepsilon_{t}$ is a white noise disturbance and the residuals from the long-run equation are used to estimate $\Delta \mu_{t} . I_{t}$ is the Heaviside indicator function such that

$$
I_{t}=\left\{\begin{array}{l}
1 \text { if } \xi_{t-1} \geq \tau \\
0 \text { if } \xi_{t-1}<\tau
\end{array}\right.
$$




\section{Table 2. TARCH and EGARCH multivariate results}

If in (4) $\xi_{t-1}=\mu_{t-1}$, then the model specification illustrated in (3) is called the threshold autoregressive (TAR) model. It allows for different coefficients of positive and negative variations. A sufficient condition for the stationarity of $\mu_{t}$ is $-2<\left(\rho_{1}, \rho_{2}\right)<0$. This means that the long-run equation is an attractor such that $\mu_{t}$ can be written as an error correction model similar to that given in (3). If $\rho_{1}=\rho_{2}$ then the adjustment is symmetric, which is a special case of (3i) and (44). Expression (3) can also contain lagged values of $\Delta \mu$. When $\mu_{t}$ is above its long-run equilibrium value, the adjustment is $\rho_{1} \mu_{t-1}$, and if $\mu_{t-1}$ is 
below its long-run equilibrium value, the adjustment is $\rho_{2} \mu_{t-1}$.

If in (4) $\xi_{t-1}=\Delta \mu_{t-1}$, then the model (3) is called the momentum threshold autoregressive (M-TAR) model. The M-TAR model allows the decay to depend on the previous period change in $\mu_{t-1}$. The value of the threshold $\tau$, in our case, will be assumed to be zero in all models.

The TAR model is designed to capture asymmetrically deep movements in the series of the deviations from the long-run equilibrium, while the M-TAR model is useful to capture the possibility of asymmetrically steep movements in the series (Enders and Granger [31]). For example, in the TAR model if $-1<\rho_{1}<\rho_{2}<0$, then the negative phase of $\mu_{t}$ will tend to be more persistent than the positive phase. On the other hand, for the M-TAR model, if for example $\left|\rho_{1}\right|<\left|\rho_{2}\right|$ the model exhibits little decay for positive $\Delta \mu_{t-1}$ but substantial decay for negative $\Delta \mu_{t-1}$. This means that increases tend to persist but decreases tend to revert quickly toward the attractor.

Finally, we can perform a number of statistical tests on the estimated coefficients (and also on the residuals) in order to ascertain the validity of the error correction model outlined in (3), and subsequently if the adjustment is symmetric or not. The relevant tests on the coefficients are $H_{0}: \rho_{1}=0$ and $H_{0}: \rho_{2}=0$, for which we obtain the sample values of the t-statistics; and $H_{0}: \rho_{1}=\rho_{2}=0$, for which we obtain the sample values of the $F$-statistic. The restriction that adjustment is symmetric, i.e. $H_{0}: \rho_{1}=\rho_{2}$, can also be tested using the usual $F$-statistic.

If the variables in the long-run equation are stationary, the usual critical values of the $t$ and $F$ distributions can be used to assess the significance level of the underlying tests. However, if these variables are integrated of first order, one can use the critical values reported by Enders and Siklos [32] to determine whether the null hypothesis of no cointegration can be rejected. If the alternative hypothesis is accepted, it is possible to test for asymmetric adjustment using the standard critical values of the $F$ distribution, since $\rho_{1}$ and $\rho_{2}$ converge to a multivariate normal distribution (Enders and Granger [31]).

Table 3 contains the results of the TAR and M-TAR models. As may be seen, for the first two tests the null hypothesis is rejected in all cases. Thus, we may proceed our analysis with the tests of symmetry and the results are that the null hypothesis is not rejected in any of the models considered. 
Table 3. TAR and M-TAR results

\section{Conclusions}

This paper presents a comparative analysis between four volatility models (TARCH, EGARCH, TAR and M-TAR) in order to ascertain their power to capture asymmetric cycles in the underlying time series. Our results indicate that while the TAR and M-TAR models do not identify the presence of asymmetry in any market, the TARCH and EGARCH models have captured asymmetry in all cases under analysis. 
Another relevant conclusion from the multivariate TARCH and EGARCH results is that the Portuguese stock market appears to reveal higher $\alpha$ values than the remaining markets, for both models. These figures imply that shocks in the Portuguese market, in the later periods, tend to have longer duration periods. This circumstance implies that the effects of shocks in earlier periods tend to linger around for a longer period than it does in other stock markets. This means that for the Portuguese stock market the effects of shocks take a longer time to dissipate.

\section{References}

[1] Fisher, L., Journal of Business, 39 (1966) 191-225.

[2] Scholes, M. and Williams, J., Journal of Financial Economics, 5 (1977) 309-327.

[3] Lo, A.W. and MacKinlay, C.A., Journal of Econometrics, 45 (1990) 181-211.

[4] Fama, E.F. and French, K.R., Journal of Financial Economics, 3 22(1) (1988) $3-25$.

[5] Conrad, J. and Kaul, G., Journal of Business, 61 (1988) 409-425.

[6] Hamao, Y., Masulis, R.W. and Ng V., The Review of Financial Studies, 3 (1990) 281-307.

[7] Wei, J., Liu, Y.J., Yang, C. and Chaung, G., Pacific-Basin Finance Journal, 3 (1995) 113-136.

[8] Mandelbrot, B., Journal of Business, 36 (1963) 394-419.

[9] Fama, E.F., Journal of Business, 38 (1965) 34-105.

[10] Nelson, D.B., Econometrica, (1991) 161-196.

[11] Plerou, V., Gopikrishnan, P., Amaral, L., Meyer, M. and Stanley, H.E., Phys. Rev. E, 60 (1999) 6519-6529.

[12] Bouchaud, J. P. and Potters, M., Physica A, 299 (2001) 60-70.

[13] Lux., T., Applied Financial Economics, 6 (1996) 463-475.

[14] Masoliver, J. and Perello, J., App. Fin., 5 (2002) 541-562.

[15] Engle, R. and Patton, A. Quantitative Finance, 1 (2001) 237-245.

[16] Pochart. B. and Bouchaud, J-P., Quantitative Finance, 2 (2002) 303-314.

[17] Muzy, J-F., Delour, J. and Bacry, E., Phys. J. B 17 (2000) 537-548.

[18] Mandelbrot, B., Fischer, A. and Calvet, L., Cowless Foundation Discussion Paper \#1164, (1997). 
[19] Bacry, E., Delour, J. and Muzy, J-F., Physical Review E, 64 (2001) 026103026106.

[20] Z. Eisler and Kertesz , J., Physica A 343C (2004) 603-622.

[21] Ross, S.A., Journal of Finance, 54 (1989) 1-17.

[22] Black, F., in Proceedings of Meetings of the Business and Economic Statistics Section, American Statistical Association (1976).

[23] Christie, A.A., Journal of Financial Economics, 10 (1982) 407-432.

[24] Engle, R.F., Financial Analysts Journal, (1993) 72-78.

[25] Zakoian, J.M., Manuscript CREST, INSEE, Paris (1990).

[26] Glosten, L.R., Jagannathan, R. and Runkle, D.E., Journal of Finance, 48 (1993) 1779-1801.

[27] Engle, R.F. and Ng, V.K., Journal of Finance, 48 (1993) 1749-1778.

[28] Sims, C.A., American Economic Review, 62 (1972) 540-552.

[29] Sims, C.A., American Economic Review, 70 (1980) 250-257.

[30] Sims, C.A., Econometrica, 48 (1980) 1-48.

[31] Enders, W. and Granger, C.W.J., Journal of Business and Economic Statistics, 16 (1988) 304-311.

[32] Enders, W. and Siklos, P.L., Journal of Business and Economic Statistics, 19 (2001) 166-77.

[33] Gjerde, O. and Saettem, F., Journal of International Financial Markets, Institutions and Money, 9 (1999) 61-74.

[34] Rapach, D.E., Journal of Economics and Business, 53 (2001) 5-26.

[35] Bala, L. and Premaratne, G., Economics Working Paper, (2003), available on: http://papers.ssrn.com/sol3/papers.cfm?abstract_id=375380. 